\title{
Huntington's disease: Molecular basis of pathology and status of current therapeutic approaches (Review)
}

\author{
WEN-JUAN HUANG, WEI-WEI CHEN and XIA ZHANG \\ Department of Neurology, Xuzhou Central Hospital, Xuzhou, Jiangsu 221009, P.R. China
}

Received May 5, 2016; Accepted July 27, 2016

DOI: $10.3892 /$ etm.2016.3566

\begin{abstract}
Huntington's disease (HD) is a frequent and incurable hereditary neurodegenerative disorder that impairs motor and cognitive functions. Mutations in huntingtin (HTT) protein, which is essential for neuronal development, lead to the development of HD. An increase in the number of CAG repeats within the $H T T$ gene, which lead to an expansion of polyglutamine tract in the resulting mutated HTT protein, which is toxic, is the causative factor of HD. Although the molecular basis of HD is known, there is no known cure for this disease other than symptomatic relief treatment approaches. The toxicity of mutHTT appears to be more detrimental to striatal medium spiny neurons, which degenerate in this disease. Therapeutic strategies addressing a reduction in the mutHTT content at the transcriptional level using zinc finger proteins and at the translational level with RNA interference and antisense oligonucleotides or promoting the proteosomal degradation of mutHTT are being studied extensively in preclinical models and also to a limited extent in clinical trials. The post-translational modification of mutHTT is another possibility that is currently being investigated for drug development. In addition to the pharmacological approaches, several lines of evidence suggested the potential therapeutic use of stem cell therapy, in particular using the patient-derived induced pluripotent stem cells, to replace the lost striatal neurons. The multi-pronged clinical investigations currently underway may identify therapies and potentially improve the quality of life for the HD patients in future.
\end{abstract}

\section{Contents}

1. Introduction

2. HTT and mutated HTT

Correspondence to: Dr Wen-Juan Huang, Department of Neurology, Xuzhou Central Hospital, 199 Jiefang South Road, Xuzhou, Jiangsu 221009, P.R. China

E-mail: ftmlb8556215@163.com

Key words: Huntington's disease, striatal medium spiny neurons, polyglutamine tract, neurodegeneration, proteosomal degradation, $\mathrm{CAG}$ repeats
3. Post-translational modifications of HTT/mutHTT

4. Therapeutic approaches

5. Conclusions

\section{Introduction}

Huntington's disease (HD) is a lethal autosomal dominant and progressive neurodegenerative disorder, that is characterized by motor, cognitive, and behavioral impairment (1). HD incidence is approximately 5-10 in 100,000 individuals worldwide (2) and encompasses psychiatric symptoms (e.g., affective disorders, suicide tendency, mania, apathy, and schizophrenia-like symptoms), cognitive defects (e.g., organizational deficit, lack of attention and motor skill learning deficits), motor impairment (e.g., chorea, rigidity, gait abnormalities, and bradykinesia), sleep disturbance, and weight loss (3).

Despite the identification of the gene that is critical for the pathogenesis of HD as huntingtin (HTT), located in the short arm of chromosome 4, >20 years ago (4), the development of effective therapies for HD are is proving to be formidable. Currently, there are no disease-modifying treatments available other than some approaches to address certain specific symptoms of HD. Onset of HD symptoms emerges usually at 35-45 years of age and varies considerably (5). HD leads to severe brain atrophy and death, with a clinical course that spans $>15-20$ years (1). Specifically, striatal medium spiny neurons (MSNs) of brain appear to be vulnerable in HD, although potentially other regions of brain can also be affected (6-8). MSNs are GABAergic neurons, are predominant in the striatum (9), and project to the substantia nigra (striatonigral) and globus pallidus (striatopallidal) (10). It has been reported that there is a significant loss of approximately $88 \%$ striatal neurons in HD patients as compared to healthy individuals, even though the precise reasons for this selective vulnerability and loss of striatal MSNs is not known $(11,12)$.

\section{HTT and mutated $H T T$}

It has been recognized that the gene encoding huntingtin (HTT), present on chromosome 4 is mutated in the exon-1 region with the resultant expansion of a CAG repeat region in this exon. This leads to enlargement of the polyglutamine (polyQ) domain within the HTT protein's N-terminus in HD patients (4). Thus, while the normal HTT gene has 
approximately $35 \mathrm{CAG}$ repeats, mutations that increase this to $\geq 40$ CAGs, lead to the development of HD with full penetrance, and individuals with 36-39 CAG repeats in HTT exhibit variability in the appearance of HD (13). Although HD can be inherited in an autosomal dominant pattern, because of the instability of the number of CAG repeats in HTT gene, this number can be different between a parent and child $(14,15)$, with a tendency to increase in the next generation. Such instability of the CAG repeats has also been noted within the same patient's brain and sperm cells, resulting in mosaicism (16). These elevated number of CAG repeats appear to cause either 'gain-of-function' or loss of function of the wild-type HTT with toxic effects, including HD-related cardiac dysfunction (17) and skeletal muscle wasting (18). Most of the mutations in muHTT disrupt its normal function, and promote several pathological protein-protein interactions resulting in neuronal loss and dysfunction in the striatum, cortex and other parts of the brain (19). Thus, mutHTT interferes with several intracellular activities through aberrant interactions as well as the accumulation of mutHTT aggregates, particularly in the cell nucleus and neurophil of the affected neurons, ultimately disrupting several cell processes, including protein degradation, mitochondrial respiration and transcription, leading to neuronal malfunction and cell death (20). Apparently the increase in CAG repeats can be as high as 1,000 in certain subsets of striatal neurons while in other brain regions the increase is much lower (21). Recent genome-wide single nucleotide polymorphism (SNP) association studies showed that MLH1 (MutL homolog 1, a DNA mismatch repair gene) and an SNP within a nuclear factor- $\kappa \mathrm{B}$ binding site in the HTT promoter may play a role in the altered onset of $\operatorname{HD}(5,22)$.

Notably, although mutations in HTT gene lead to the pathogenesis of HD, this is an essential gene for normal neuronal development as knockout of the HTT gene $\left(\mathrm{Hdh}^{-/}\right)$is lethal in embryonic mice $(23,24)$. There is significant neurodegeneration and motor and cognitive abnormalities even with a single allele deletion $\left(\mathrm{Hdh}^{+/}\right)$in mice (23). Even conditional Hdh deletion in adult mice in the neurons of the forebrain and to a small extent in cerebellum leads to the development of progressive neurodegeneration (25). HTT is a $350-\mathrm{kDa}$ protein with dynamic subcellular localization in nuclei, endoplasmic reticulum, Golgi apparatus and endosomes and is known to have functions in the regulation of cell cycle and cell division $(26,27)$. HTT is also found in axonal processes and synapses in association with microtubules, caveolae and synaptosomes (28).

\section{Post-translational modifications of HTT/mutHTT}

Post-translational modifications of the HTT protein play an important role in the pathogenesis of HD (Fig. 1). For example, mutHTT is prone to aggregate in neurons, which is suspected to be part of the underlying causes of HD. Although mutHTT is ubiquitinylated, its clearance by the proteosomal system is impaired leading to accumulation of the aggregates (29). HTT is also likely modified by phosphorylation, SUMOylation, acetylation and palmitoylation and these post-translational modifications are important in proper protein-protein interactions of HTT, which can be significantly altered by mutations and polyQ additions (30). Histone acetyltransferase (HAT) enzymes CBP and PCAF were found to be inactivated by mutHTT through protein-protein interactions, leading to transcriptional and chromatin remodeling deregulation and contributing to the pathogenesis of HD (31). It has been suggested that post-translational modifications be exploited for therapeutic purposes to enhance the clearance of mutHTT. Thus, acetylation of the lysine residue K444 in mutHTT enhanced its clearance via autophagosomes (32), whereas the phosphorylation of mutHTT at serine 431 and 432 altered the toxicity and accumulation of mutHTT (33). Phosphorylation of serine residues 13 and 16 reduced its toxicity of mutHTT in vivo (34), whereas phosphorylation at serine 421 restored the ability of mutHTT to promote axonal vesicular transport and brain-derived neurotrophic factor release (35).

\section{Therapeutic approaches}

Therapies that address mutHTT modification and degradation. Due to the interaction-mediated inhibitory effects of mutHTT on HAT enzymes, certain inhibitors of histone deacetylases (HDAC), in particular HDAC4, have been examined for their protective effects in some models of HD. The findings showed that these inhibitors were able to reduce the aggregation of mutHTT and also rescue the neuronal and corticostriatal synaptic function $(36,37)$. Notably, acetylation of mutHTT marks it for ubiquitinylation and subsequent proteosomal degradation and there is a general decline in chromosomal and protein acetylation in HD. Thus, inhibition of HDACs, which sustains an elevated level of protein acetylation, can lead to an increased acetylation status of mutHTT (38). Inhibitors of other deacetylase enzymes such as sirtuin 1, and selisistat are shown to curtail the mutHTT-induced pathology in several model systems (39) and proved to be safe and tolerable in recent phase $1 \mathrm{~B}$ clinical trials (40). Promotion of the proteolytic breakdown of mHTT through activation of the ubiquitin- proteasome- autophagy system is another pharmacological approach that is being explored (41). Thus, promoting autophagy by inhibiting mTOR with rapamycin, was shown to improve phenotypes in HD models in Drosophila and mouse (38) and similar effects were observed with other autophagy-promoting agents (42). Thus, enhancing autophagy to degrade mutHTT is a viable and important strategy towards HD therapy (Fig. 2).

Considering that selective modulation of phosphorylation of serine residues can be exploited to modulate mutHTT activity, small molecule kinase inhibitors are being tested, even though their selectivity is being investigated (43). Inasmuch as improper folding and aggregation of mutHTT is central to the pathogenesis of $\mathrm{HD}$, attempts are being made to devise cell-permeable chaperones, such as TCP1-ring complex and ApiCCT1 to selectively prevent the aggregation of mutHTT and associated toxicity in neuronal cells $(44,45)$.

Therapies addressing signaling pathways. Another important HD pathology-associated change is in the cyclic AMP (cAMP) signaling (46) and aberrant transcription of genes regulated by the cAMP response element (CRE) (47). Inhibition of phosphodiesterase (PDE) 10A, which regulates cAMP and cyclic guanosine monophosphate signaling, and is mostly expressed in the MSNs of striatum (48) is shown to be beneficial against 


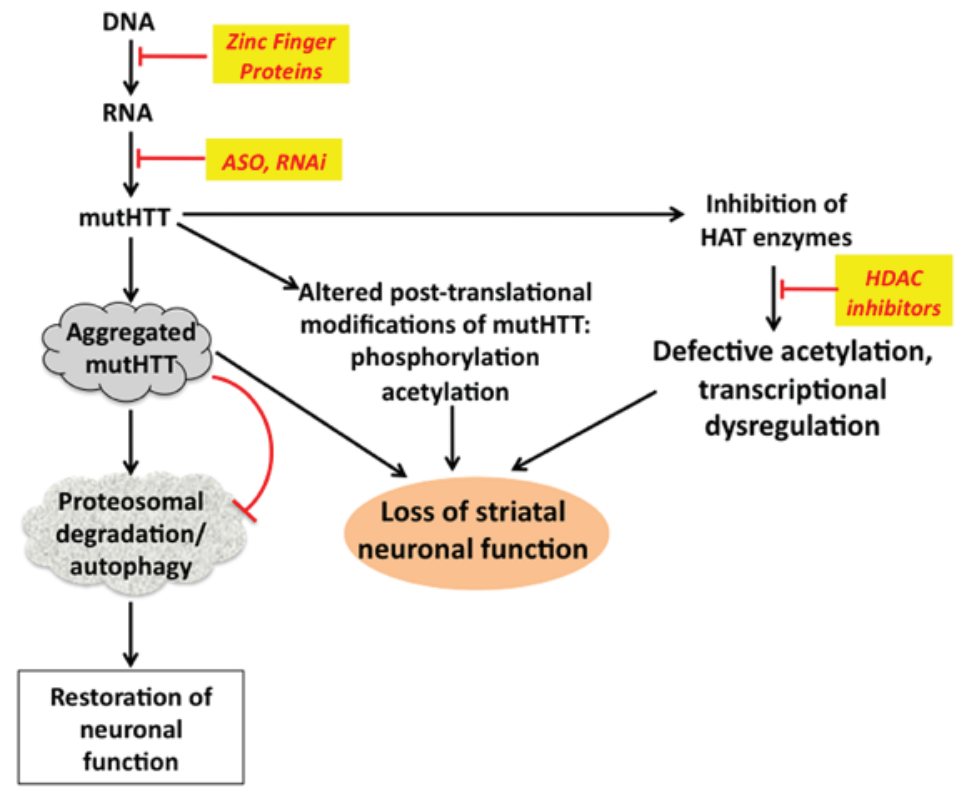

Figure 1. Potential pathological molecular events in Huntington's disease and possible therapeutic interventions. ASO, antisense oligonucleotides; HTT, huntingtin; HDAC, histone deacetylases; HAT, histone acetyltransferase.

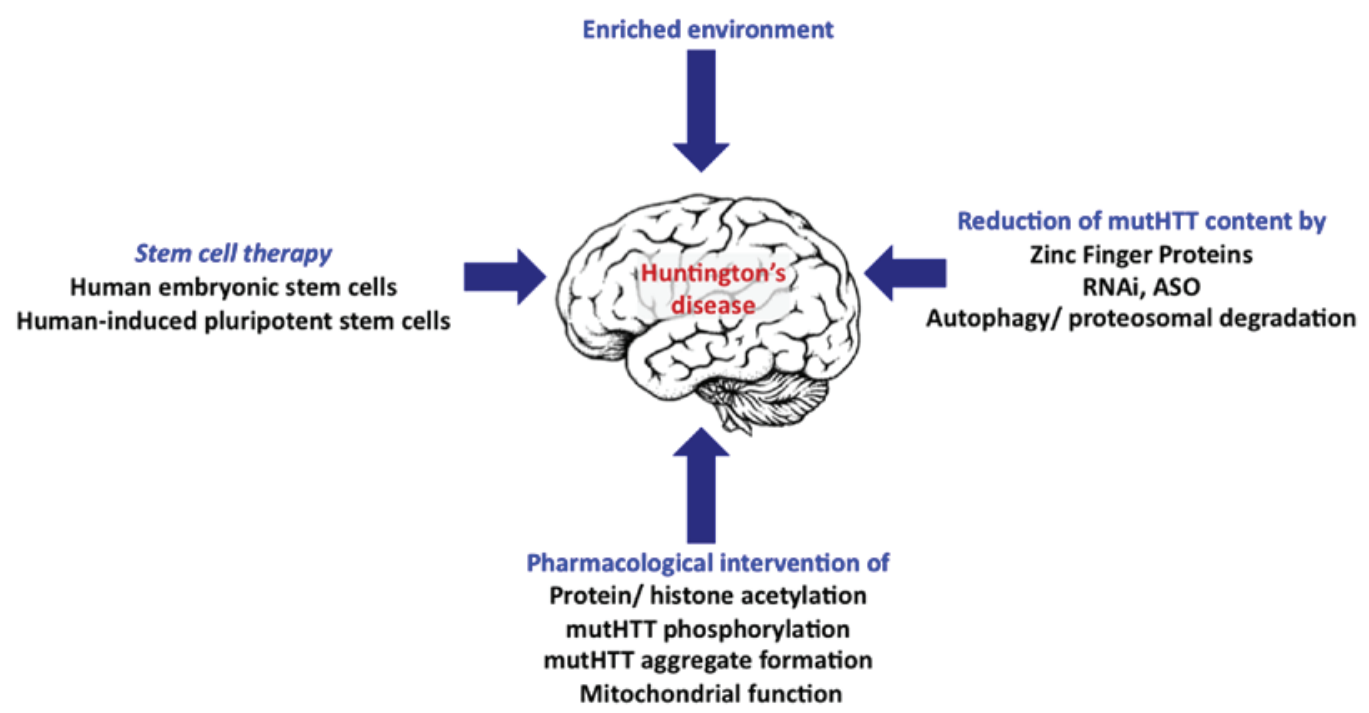

Figure 2. Emerging therapies for Huntington's disease. ASO, antisense oligonucleotides; HTT, huntingtin.

HD, via restoration of CRE-mediated gene expression (49). Speciffically, PDE10A inhibitor-based clinical trials in HD patients are currently addressing the efficacy and motor functional endpoints (50).

An important signaling pathway that is hyperactive and contributes to the pathology of HD is MAPK signaling (51). Specifically, overactive c-Jun N-terminal kinase likely leads to dysregulated axonal transport (52) and hyperactive p38 may cause NMDA receptor-mediated excitotoxicity (53). Thus, the overexpression of MKP-1, a negative modulator of MAPKs, was shown to prevent against mutHTT-mediated neuronal dysfunction in several models of HD (54). Similarly, inhibition of MLK-2 was retarded mutHTT mediated-toxicity (55). NMDA receptor-mediated excitotoxicity, has been suspected to be an important contributor to HD pathogenesis and quinolinic acid, an endogenous degradation product of tryptophan, is a known NMDA receptor agonist. In the pathway of tryptophan catabolism, kynurenine monooxygenase (KMO) activity determines the balance between the neuroprotective kynurenic acid and neurotoxic quinolinic acid. Post-mortem examination of brains from HD patients revealed that there is an increase in quinolinic acid and decrease in kynurenic acid. Treatment of HD animal models with an inhibitor of KMO led to elevated kynurenic acid, as well as improved survival and striatal neuron function. Recent studies reported an improved KMO inhibitor, CHDI-340246, which acts only peripherally and elevates kynurenine and kynurenic acid in HD rodent and non-human primate models, and protects from neuronal loss and dysfunction $(50,56)$.

Therapies that decrease mutHTT content. Reducing the content of mutHTT by inhibiting gene transcription, mRNA 
translation or promoting the breakdown of mRNA coding for HTT, may reduce any associated downstream damaging effects of mutHTT, which otherwise lead to the pathogenesis of HD. However, considering that loss of HTT protein, even conditionally, led to neurodegeneration, caution must be exercised to employ procedures that suppress HTT completely. It is more prudent to selectively target HTT genes that harbor excessive CAG repeats, and not normal HTT gene (Figs. 1 and 2).

Inhibition of transcription by zinc finger proteins (ZFPs) were used to reduce the transcription from the HTT gene. ZFPs can be designed to allow specific binding to selected DNA sequences, and are fused to a transcriptional repressor domain, in order that the gene to which these ZFPs bind, is not expressed, and thus the corresponding protein production is blocked (57). Using ZFPs it has been observed that the proximity of the CAG repeat to the 5' end of the HTT gene confers selectivity over other genes containing poly-CAG sequences for targeting with viral vectors for delivering the ZFPs (58). Such as approach has been used successfully utilised in mouse model of HD with the resultant decease in pathological motor manifestations $(59,60)$. ZFPs have been employed to deliver DNA nucleases to the target sequences, in a way that excessive CAG repeats are excised from HTT genes, thus raising the prospect of gene therapy for HD (61).

Another approach to lower the expression of mutHTT is to target the corresponding mRNA with specific antisense oligonucleotides (ASOs), which are single-stranded DNA oligonucleotides, and bind to complimentary mRNA sequences via base-pairing and lead to the degradation of the mRNA by RNAse H. Previous findings have shown that intraventricular infusion of mutHTT targeting chemically modified ASOs in three separate HD mouse models was successful in reducing HTT mRNA by $60 \%$ and HTT protein by $>80 \%$ reduction, in a dose-dependent manner. These changes were accompanied by delayed mutHTT aggregation and improved motor performance on a rotarod test. This ASO-induced restoration of normal functionality was sustained even after the infused ASOs were removed, indicating that there was a restoration and recovery of the neurons rendered dysfunctional due to mutHTT (62-64). An advantage of use of ASO is its broad distribution into different brain regions following intraventricular infusion. Inasmuch as mutHTT synthesis is rather ubiquitous, a wider distribution of ASOs is useful in targeting mutHTT expression and thus curtailing its deleterious effects (62). Intrathecal infusion of ASOs for 21 days in non-human primates led to a sustained decrease by approximately $50 \%$ in mutHTT mRNA levels in frontal cortex, occipital cortex (68\%), and spinal cord, indicating the possible application of ASOs for human situation (62).

Recent advancements in gene silencing efficiency as well as sustained long-term effects of RNAi agents have overtaken the ZFPs and ASOs, despite their therapeutic potential (65). RNAi techniques were successfully employed to reduce HTT mRNA and protein in in vitro models of HD (66). Subsequent, in vivo studies in HD transgenic mice employing AAV-based delivery of shRNA targeting HTT via single bilateral injections into the striatum, revealed a significant reduction in mutHTT mRNA and protein levels, mutHTT aggregates and marked improvement in behavioral and motor performance parameters (67). This study was followed by several other in vivo studies using RNAi approach with improvements in many other HD-associated pathologies (68). Although many of these initial studies employed pre-symptomatic animal models of HD, subsequent studies showed that RNAi approaches also reduced the number of mutHTT inclusions and significantly improved striatal functionality and motor performance (69). Despite the success of these gene expression approaches, clinical application of these methods is not yet feasible and much refinement needs to be attained in these technologies.

In addition to the abovementioned approaches, stem cell-based therapies, in particular, using the patient-specific iPSCs are being developed and are promising to combat HD (Fig. 2) (70).

\section{Conclusions}

HD is a hereditary neurodegenerative disorder that impairs motor and cognitive functions, by targeting striatal MSNs, with no known cure. MutHTT protein, with an expansion of polyQ tract is toxic to neurons and is the causative factor of HD. Therapeutic strategies addressing a reduction in the mutHTT content at the genome, mRNA or protein degradation level and post-tranlational modification of mutHTT are being studied in preclinical models and in clinical trials. Besides the pharmacological approaches, the use of stem cell therapy, to replace the lost striatal neurons, is also being examined. These multiple clinical investigations are promising to identify therapies that may improve the quality of life for HD patients in future.

\section{References}

1. Ross CA, Aylward EH, Wild EJ, Langbehn DR, Long JD, Warner JH, Scahill RI, Leavitt BR, Stout JC, Paulsen JS, et al: Huntington disease: Natural history, biomarkers and prospects for therapeutics. Nat Rev Neurol 10: 204-216, 2014.

2. Pringsheim T, Wiltshire K, Day L, Dykeman J, Steeves T and Jette N: The incidence and prevalence of Huntington's disease: A systematic review and meta-analysis. Mov Disord 27: 1083-1091, 2012.

3. Bates G, Harper P and Jones L (eds). Huntington's disease. Oxford University Press, New York, NY, 2002.

4. MacDonald M; The Huntington's Disease Collaborative Research Group: A novel gene containing a trinucleotide repeat that is expanded and unstable on Huntington's disease chromosomes. Cell 72: 971-983, 1993.

5. Genetic Modifiers of Huntington's Disease (GeM-HD) Consortium: Identification of genetic factors that modify clinical onset of Huntington's disease. Cell 162: 516-526, 2015.

6. Graveland GA, Williams RS and DiFiglia M: Evidence for degenerative and regenerative changes in neostriatal spiny neurons in Huntington's disease. Science 227: 770-773, 1985.

7. Mann DM, Oliver R and Snowden JS: The topographic distribution of brain atrophy in Huntington's disease and progressive supranuclear palsy. Acta Neuropathol 85: 553-559, 1993.

8. Rosas HD, Koroshetz WJ, Chen YI, Skeuse C, Vangel M, Cudkowicz ME, Caplan K, Marek K, Seidman LJ, Makris N, et al: Evidence for more widespread cerebral pathology in early HD: An MRI-based morphometric analysis. Neurology 60: 1615-1620, 2003.

9. Kemp JM and Powell TP: The structure of the caudate nucleus of the cat: Light and electron microscopy. Philos Trans R Soc Lond B Biol Sci 262: 383-401, 1971.

10. Parent A, Bouchard C and Smith Y: The striatopallidal and striatonigral projections: Two distinct fiber systems in primate. Brain Res 303: 385-390, 1984.

11. Heinsen H, Strik M, Bauer M, Luther K, Ulmar G, Gangnus D, Jungkunz G, Eisenmenger W and Götz M: Cortical and striatal neurone number in Huntington's disease. Acta Neuropathol 88: 320-333, 1994. 
12. Vonsattel JP and DiFiglia M: Huntington disease. J Neuropathol Exp Neurol 57: 369-384, 1998.

13. Myers RH: Huntington's disease genetics. NeuroRx 1: 255-262, 2004.

14. Duyao M, Ambrose C, Myers R, Novelletto A, Persichetti F, Frontali M, Folstein S, Ross C, Franz M, Abbott M, et al: Trinucleotide repeat length instability and age of onset in Huntington's disease. Nat Genet 4: 387-392, 1993.

15. Trottier Y, Biancalana V and Mandel JL: Instability of CAG repeats in Huntington's disease: Relation to parental transmission and age of onset. J Med Genet 31: 377-382, 1994.

16. Telenius H, Kremer B, Goldberg YP, Theilmann J, Andrew SE, Zeisler J, Adam S, Greenberg C, Ives EJ, Clarke LA, et al: Somatic and gonadal mosaicism of the Huntington disease gene CAG repeat in brain and sperm. Nat Genet 6: 409-414, 1994.

17. Zielonka D, Piotrowska I and Mielcarek M: Cardiac dysfunction in huntington's disease. Exp Clin Cardiol 20: 2547-2554, 2014.

18. Zielonka D, Piotrowska I, Marcinkowski JT and Mielcarek M: Skeletal muscle pathology in Huntington's disease. Front Physiol 5: 380, 2014

19. Imarisio S, Carmichael J, Korolchuk V, Chen CW, Saiki S, Rose C, Krishna G, Davies JE, Ttofi E, Underwood BR, et al: Huntington's disease: From pathology and genetics to potential therapies. Biochem J 412: 191-209, 2008.

20. Valor LM: Transcription, epigenetics and ameliorative strategies in Huntington's Disease: A genome-wide perspective. Mol Neurobiol 51: 406-423, 2015

21. Kennedy L, Evans E, Chen CM, Craven L, Detloff PJ, Ennis M and Shelbourne PF: Dramatic tissue-specific mutation length increases are an early molecular event in Huntington disease pathogenesis. Hum Mol Genet 12: 3359-3367, 2003.

22. Bečanović K, Nørremølle A, Neal SJ, Kay C, Collins JA, Arenillas D, Lilja T, Gaudenzi G, Manoharan S, Doty CN, et al REGISTRY Investigators of the European Huntington's Disease Network: A SNP in the HTT promoter alters NF- $\kappa \mathrm{B}$ binding and is a bidirectional genetic modifier of Huntington disease. Nat Neurosci 18: 807-816, 2015.

23. Nasir J, Floresco SB, O'Kusky JR, Diewert VM, Richman JM, Zeisler J, Borowski A, Marth JD, Phillips AG and Hayden MR: Targeted disruption of the Huntington's disease gene results in embryonic lethality and behavioral and morphological changes in heterozygotes. Cell 81: 811-823, 1995.

24. Zeitlin S, Liu JP, Chapman DL, Papaioannou VE and Efstratiadis A: Increased apoptosis and early embryonic lethality in mice nullizygous for the Huntington's disease gene homologue. Nat Genet 11: 155-163, 1995.

25. Dragatsis I, Levine MS and Zeitlin S: Inactivation of Hdh in the brain and testis results in progressive neurodegeneration and sterility in mice. Nat Genet 26: 300-306, 2000.

26. Hoffner G, Kahlem P and Djian P: Perinuclear localization of huntingtin as a consequence of its binding to microtubules through an interaction with beta-tubulin: Relevance to Huntington's disease. J Cell Sci 115: 941-948, 2002.

27. Godin JD, Colombo K, Molina-Calavita M, Keryer G, Zala D, Charrin BC, Dietrich P, Volvert ML, Guillemot F, Dragatsis I, et al: Huntingtin is required for mitotic spindle orientation and mammalian neurogenesis. Neuron 67: 392-406, 2010.

28. DiFiglia M, Sena-Esteves M, Chase K, Sapp E, Pfister E, Sass M, Yoder J, Reeves P, Pandey RK, Rajeev KG, et al: Therapeutic silencing of mutant huntingtin with siRNA attenuates striatal and cortical neuropathology and behavioral deficits. Proc Natl Acad Sci USA 104: 17204-17209, 2007.

29. Arrasate M and Finkbeiner S: Protein aggregates in Huntington's disease. Exp Neurol 238: 1-11, 2012

30. Ehrnhoefer DE, Sutton L and Hayden MR: Small changes, big impact: Posttranslational modifications and function of huntingtin in Huntington disease. Neuroscientist 17: 475-492, 2011.

31. Zielonka D, Mielcarek M and Landwehrmeyer GB: Update on Huntington's disease: Advances in care and emerging therapeutic options. Parkinsonism Relat Disord 21: 169-178, 2015.

32. Jeong H, Then F, Melia TJ Jr, Mazzulli JR, Cui L, Savas JN, Voisine C, Paganetti P, Tanese N, Hart AC, et al: Acetylation targets mutant huntingtin to autophagosomes for degradation. Cell 137: 60-72, 2009.

33. Dong G, Callegari E, Gloeckner CJ, Ueffing M and Wang H: Mass spectrometric identification of novel posttranslational modification sites in Huntingtin. Proteomics 12: 2060-2064, 2012.
34. Gu X, Greiner ER, Mishra R, Kodali R, Osmand A, Finkbeiner S, Steffan JS, Thompson LM, Wetzel R and Yang XW: Serines 13 and 16 are critical determinants of full-length human mutant huntingtin induced disease pathogenesis in HD mice. Neuron 64: 828-840, 2009

35. Zala D, Colin E, Rangone H, Liot G, Humbert S and Saudou F: Phosphorylation of mutant huntingtin at S421 restores anterograde and retrograde transport in neurons. Hum Mol Genet 17: 3837-3846, 2008.

36. Venuto CS, McGarry A, Ma Q and Kieburtz K: Pharmacologic approaches to the treatment of Huntington's disease. Mov Disord 27: 31-41, 2012.

37. Mielcarek M, Benn CL, Franklin SA, Smith DL, Woodman B, Marks PA and Bates GP: SAHA decreases HDAC 2 and 4 levels in vivo and improves molecular phenotypes in the R6/2 mouse model of Huntington's disease. PLoS One 6: e27746, 2011.

38. Ravikumar B, Vacher C, Berger Z, Davies JE, Luo S, Oroz LG, Scaravilli F, Easton DF, Duden R, O'Kane CJ, et al: Inhibition of mTOR induces autophagy and reduces toxicity of polyglutamine expansions in fly and mouse models of Huntington disease. Nat Genet 36: 585-595, 2004.

39. Smith MR, Syed A, Lukacsovich T, Purcell J, Barbaro BA, Worthge SA, Wei SR, Pollio G, Magnoni L, Scali C, et al: A potent and selective Sirtuin 1 inhibitor alleviates pathology in multiple animal and cell models of Huntington's disease. Hum Mol Genet 23: 2995-3007, 2014.

40. Reilmann R, Squitieri F, Priller J, Saft C, Mariotti C, Suessmuth S, Nemeth A, Tabrizi S, Quarrell O, Craufurd D, et al: Safety and tolerability of selisistat for the treatment of huntington's disease: Results from a randomized, double-blind, placebo-controlled phase II trial. Neurology (Suppl 10): 82: S47.004, 2014.

41. Labbadia J and Morimoto RI: Huntington's disease: Underlying molecular mechanisms and emerging concepts. Trends Biochem Sci 38: 378-385, 2013

42. Renna M, Jimenez-Sanchez M, Sarkar S and Rubinsztein DC: Chemical inducers of autophagy that enhance the clearance of mutant proteins in neurodegenerative diseases. J Biol Chem 285: 11061-11067, 2010.

43. Atwal RS, Desmond CR, Caron N, Maiuri T, Xia J, Sipione S and Truant R: Kinase inhibitors modulate huntingtin cell localization and toxicity. Nat Chem Biol 7: 453-460, 2011.

44. Tam S, Geller R, Spiess C and Frydman J: The chaperonin TRiC controls polyglutamine aggregation and toxicity through subunit-specific interactions. Nat Cell Biol 8: 1155-1162, 2006.

45. Sontag EM, Joachimiak LA, Tan Z, Tomlinson A, Housman DE, Glabe CG, Potkin SG, Frydman J and Thompson LM: Exogenous delivery of chaperonin subunit fragment ApiCCT1 modulates mutant Huntingtin cellular phenotypes. Proc Natl Acad Sci USA 110: 3077-3082, 2013.

46. Gines S, Seong IS, Fossale E, Ivanova E, Trettel F, Gusella JF, Wheeler VC, Persichetti F and MacDonald ME: Specific progressive cAMP reduction implicates energy deficit in presymptomatic Huntington's disease knock-in mice. Hum Mol Genet 12: 497-508, 2003.

47. Sugars KL, Brown R, Cook LJ, Swartz J and Rubinsztein DC: Decreased cAMP response element-mediated transcription: An early event in exon 1 and full-length cell models of Huntington's disease that contributes to polyglutamine pathogenesis. J Biol Chem 279: 4988-4999, 2004

48. Coskran TM, Morton D, Menniti FS, Adamowicz WO, Kleiman RJ, Ryan AM, Strick CA, Schmidt CJ and Stephenson DT: Immunohistochemical localization of phosphodiesterase $10 \mathrm{~A}$ in multiple mammalian species. J Histochem Cytochem 54: 1205-1213, 2006.

49. Kleiman RJ, Kimmel LH, Bove SE, Lanz TA, Harms JF, Romegialli A, Miller KS, Willis A, des Etages S, Kuhn M, et al: Chronic suppression of phosphodiesterase 10A alters striatal expression of genes responsible for neurotransmitter synthesis, neurotransmission, and signaling pathways implicated in Huntington's disease. J Pharmacol Exp Ther 336: 64-76, 2011.

50. Wild EJ and Tabrizi SJ: Targets for future clinical trials in Huntington's disease: What's in the pipeline? Mov Disord 29: 1434-1445, 2014.

51. Gianfriddo M, Melani A, Turchi D, Giovannini MG and Pedata F: Adenosine and glutamate extracellular concentrations and mitogen-activated protein kinases in the striatum of Huntington transgenic mice. Selective antagonism of adenosine A2A receptors reduces transmitter outflow. Neurobiol Dis 17: $77-88,2004$ 
52. Morfini GA, You YM, Pollema SL, Kaminska A, Liu K, Yoshioka K, Björkblom B, Coffey ET, Bagnato C, Han D, et al: Pathogenic huntingtin inhibits fast axonal transport by activating JNK3 and phosphorylating kinesin. Nat Neurosci 12: 864-871, 2009.

53. Fan J, Gladding CM, Wang L, Zhang LY, Kaufman AM, Milnerwood AJ and Raymond LA: P38 MAPK is involved in enhanced NMDA receptor-dependent excitotoxicity in YAC transgenic mouse model of Huntington disease. Neurobiol Dis 45: 999-1009, 2012

54. Taylor DM, Moser R, Régulier E, Breuillaud L, Dixon M, Beesen AA, Elliston L, Silva Santos MF, Kim J, Jones L, et al: MAP kinase phosphatase 1 (MKP-1/DUSP1) is neuroprotective in Huntington's disease via additive effects of JNK and p38 inhibition. J Neurosci 33: 2313-2325, 2013.

55. Apostol BL, Simmons DA, Zuccato C, Illes K, Pallos J, Casale M, Conforti P, Ramos C, Roarke M, Kathuria S, et al: CEP-1347 reduces mutant huntingtin-associated neurotoxicity and restores BDNF levels in R6/2 mice. Mol Cell Neurosci 39: $8-20,2008$

56. Mrzljak L: A05 targeting kmo: Basic understanding and gaps. J Neurol Neurosurg Psychiatry 85: A2, 2014.

57. Papworth M, Kolasinska P and Minczuk M: Designer zinc-finger proteins and their applications. Gene 366: 27-38, 2006.

58. Jiang H, Sun YM, Hao Y, Yan YP, Chen K, Xin SH, Tang YP, Li XH, Jun T, Chen YY, Liu ZJ, Wang CR, Li H, Pei Z, Shang HF, Zhang BR, Gu WH, Wu ZY, Tang BS and Burgunder JM: Huntingtin gene CAG repeat numbers in Chinese patients with Huntington's disease and controls. Eur J Neurol 21: 637-642, 2014.

59. Garriga-Canut M, Agustín-Pavón C, Herrmann F, Sánchez A Dierssen M, Fillat $C$ and Isalan M: Synthetic zinc finger repressors reduce mutant huntingtin expression in the brain of R6/2 mice. Proc Natl Acad Sci USA 109: E3136-E3145, 2012.

60. Zeitler J, Pearl JR, Froelich S, Yu Q, Paschon DE, Miller JC, Marlen K, Guschin D, Narayanan A, Zhang L, et al: Allelespecific repression of mutant Huntingtin expression by engineered zinc finger transcriptional repressors as a potential therapy for Huntington's disease. PNAS 108: 7052-7057, 2011.

61. Li H, Haurigot V, Doyon Y, Li T, Wong SY, Bhagwat AS Malani N, Anguela XM, Sharma R, Ivanciu L, et al: In vivo genome editing restores haemostasis in a mouse model of haemophilia. Nature 475: 217-221, 2011.
62. Kordasiewicz HB, Stanek LM, Wancewicz EV, Mazur C, McAlonis MM, Pytel KA, Artates JW, Weiss A, Cheng SH, Shihabuddin LS, et al: Sustained therapeutic reversal of Huntington's disease by transient repression of huntingtin synthesis. Neuron 74: 1031-1044, 2012.

63. Lu XH and Yang XW: 'Huntingtin holiday': Progress toward an antisense therapy for Huntington's disease. Neuron 74: 964-966, 2012.

64. Carroll JB, Warby SC, Southwell AL, Doty CN, Greenlee S, Skotte N, Hung G, Bennett CF, Freier SM and Hayden MR: Potent and selective antisense oligonucleotides targeting single-nucleotide polymorphisms in the Huntington disease gene/allele-specific silencing of mutant huntingtin. Mol Ther 19: 2178-2185, 2011

65. Miyagishi M, Hayashi M and Taira K: Comparison of the suppressive effects of antisense oligonucleotides and siRNAs directed against the same targets in mammalian cells. Antisense Nucleic Acid Drug Dev 13: 1-7, 2003.

66. Chen ZJ, Kren BT, Wong PY, Low WC and Steer CJ: Sleeping Beauty-mediated down-regulation of huntingtin expression by RNA interference. Biochem Biophys Res Commun 329: 646-652, 2005

67. Harper SQ, Staber PD, He X, Eliason SL, Martins IH, Mao Q, Yang L, Kotin RM, Paulson HL and Davidson BL: RNA interference improves motor and neuropathological abnormalities in a Huntington's disease mouse model. Proc Natl Acad Sci USA 102: $5820-5825,2005$

68. Godinho BM, Malhotra M, O'Driscoll CM and Cryan JF Delivering a disease-modifying treatment for Huntington's disease. Drug Discov Today 20: 50-64, 2015.

69. Drouet V, Perrin V, Hassig R, Dufour N, Auregan G, Alves S, Bonvento G, Brouillet E, Luthi-Carter R, Hantraye P, et al: Sustained effects of nonallele-specific Huntingtin silencing. Ann Neurol 65: 276-285, 2009.

70. Golas MM and Sander B: Use of human stem cells in Huntington disease modeling and translational research. Exp Neurol 278: 76-90, 2016. 